\title{
The extracellular-regulated protein kinase 5 (ERK5) enhances metastatic burden in triple-negative breast cancer through focal adhesion protein kinase (FAK)-mediated regulation of cell adhesion
}

\author{
Qiuping $\mathrm{Xu}^{1} \cdot$ Jingwei Zhang ${ }^{1} \cdot$ Brian A. Telfer $^{2} \cdot \mathrm{Hao}_{\mathrm{Zhang}^{3}} \cdot \mathrm{Nisha} \mathrm{Ali}^{4} \cdot$ Fuhui Chen $^{1} \cdot$ Blanca Risa $^{1}$. \\ Adam J. Pearson ${ }^{1} \cdot$ Wei Zhang ${ }^{1} \cdot$ Katherine G. Finegan $\mathbb{B}^{2} \cdot$ Ahmet Ucar $^{1} \cdot$ Emanuele Giurisato $\mathbb{C}^{1,5} \cdot$ \\ Cathy Tournier $\mathbb{D I}^{1}$
}

Received: 21 April 2020 / Revised: 23 March 2021 / Accepted: 14 April 2021 / Published online: 12 May 2021

(c) The Author(s) 2021. This article is published with open access

\begin{abstract}
There is overwhelming clinical evidence that the extracellular-regulated protein kinase 5 (ERK5) is significantly dysregulated in human breast cancer. However, there is no definite understanding of the requirement of ERK5 in tumor growth and metastasis due to very limited characterization of the pathway in disease models. In this study, we report that a high level of ERK5 is a predictive marker of metastatic breast cancer. Mechanistically, our in vitro data revealed that ERK5 was critical for maintaining the invasive capability of triple-negative breast cancer (TNBC) cells through focal adhesion protein kinase (FAK) activation. Specifically, we found that phosphorylation of FAK at Tyr397 was controlled by a kinaseindependent function of ERK5. Accordingly, silencing ERK5 in mammary tumor grafts impaired FAK phosphorylation at Tyr397 and suppressed TNBC cell metastasis to the lung without preventing tumor growth. Collectively, these results establish a functional relationship between ERK5 and FAK signaling in promoting malignancy. Thus, targeting the oncogenic ERK5-FAK axis represents a promising therapeutic strategy for breast cancer exhibiting aggressive clinical behavior.
\end{abstract}

Supplementary information The online version contains supplementary material available at https://doi.org/10.1038/s41388021-01798-2.

Cathy Tournier

cathy.tournier@manchester.ac.uk

1 Division of Cancer Sciences, School of Medical Sciences, Faculty of Biology, Medicine and Health, University of Manchester, Manchester, UK

2 Division of Pharmacy and Optometry, School of Health Sciences, Faculty of Biology, Medicine and Health, University of Manchester, Manchester, UK

3 State Key Laboratory of Biocontrol, School of Life Sciences, Sun Yat-sen University, Guangzhou, China

4 Manchester University NHS FT, Wythenshawe hospital, Manchester, UK

5 Department of Biotechnology Chemistry and Pharmacy, University of Siena, Siena, Italy

\section{Introduction}

The overall survival of breast cancer patients has increased quite remarkably over the past few decades as a consequence of the introduction of early detection programs, but also due to the utilization of improved treatment protocols based on molecular and genomic diagnostics [1]. In particular, patterns of expression of the estrogen receptor (ER), progesterone receptor (PR) and human epidermal growth factor receptor 2 (HER2) constitute effective predictive markers for potential responses to endocrine treatment, the humanized monoclonal anti-HER2 antibody trastuzumab (Herceptin ${ }^{\circledR}$ ) and the small protein tyrosine kinase inhibitor lapatinib [2, 3]. In spite of these marked therapeutic advances, the most aggressive basal-like tumors, which largely overlap with the triple ER, PR and HER2 negative subtype of breast cancer (TNBC), continue to present a significant clinical challenge due to the limited availability of treatment options $[2,3]$. Notably, regardless of the combination chemotherapy regimen given, many responses in the metastatic setting are short, with a median overall survival being of less than 2 years [3]. Therefore, the 
identification of relevant biomarkers has become of paramount importance for guiding the future development of efficacious targeted therapies to improve TNBC patient outcome.

Signal transduction via mitogen-activated protein kinases (MAPK) are frequently dysregulated in many types of cancer. In particular, there is clinical evidence that overexpression and hyperphosphorylation of the extracellularregulated protein kinase 5 (ERK5) are associated with overall poor survival rates of breast cancer patients and resistance to chemotherapy [4-7]. Accordingly, mammary graft studies, using human (MDA-MB-231) or murine (4T1) breast cancer cell lines that mirror the molecular subtype of TNBC, demonstrated that ERK5 knockdown suppressed metastasis [8-10]. Interestingly, mice transplanted with MDA-MB-231 cells in which ERK5 was silenced exhibited a decreased number of circulating tumor cells in the blood compared with those transplanted with wild type MDA-MB-231 cells [9]. Collectively these observations in murine models added weight to the argument, from the analysis of patient tumor samples, that ERK5 targeting represented a valid strategy against metastatic breast cancer.

In contrast, there is no clarity in understanding the mechanism by which ERK5 influences metastasis-related tumor cell function. For example, ERK5 could function via rearranging the epithelial phenotype of breast cancer cells to acquire migratory and invasive characteristics [9-11]. This has been disputed by other studies which suggested that ERK5 attenuated breast cancer cell motility and suppressed mammary tumor metastasis through negative regulation of mesenchymal markers [12, 13]. Additionally, ERK5 was found to be a target of the focal adhesion protein kinase (FAK) in breast cancer cell adhesion signaling [14]. However, a functional relationship between ERK5 and FAK signaling in the pathogenesis of metastatic breast cancer has never been fully investigated in vivo. Furthermore, the precise mechanistic interaction between ERK5 and FAK remains unclear. In particular, while ERK5 was initially found to be a target of FAK [14], other studies demonstrated that ERK5 promoted breast cancer cell proliferation [15], adhesion-dependent survival [16], and melanoma cell invasiveness [17] through FAK phosphorylation at Ser910.

In this study, we have utilized the triple-negative MDAMB-231 breast cancer line and selective short hairpin RNA (shRNA) knockdown strategy to assess the cellular function under the control of ERK5 that influences the course of breast tumor progression and metastasis. The demonstration that ERK5 was required for FAK phosphorylation at Tyr397 and breast cancer metastasis identifies a previously untargeted signaling axis implicated in tumor cell migration for distant metastasis formation.

\section{Results}

\section{High ERK5 expression serves as a potentially important prognostic indicator of breast cancer malignancy and poor prognosis}

Initially, we investigated the clinical significance of ERK5 in breast cancer using the Kaplan-Meier Plotter database tool (kmplot.com). We found that the level of ERK5 expression inversely correlated with distant metastasis-free survival across all subtypes (hazard ratio $=1.3, p$ value $=$ 0.01; Fig. 1). The association of ERK5 expression with the metastatic spread of the disease was statistically significant in later stage breast cancer (all hazard ratios $\geq 1.5, p$ value $=$ 0.3 for grade 1 compared with $<0.02$ for grades 2 and 3 ). In parallel, the analysis of a large transcriptomic dataset of breast tumors from The Cancer Genome Atlas (TCGA) [18] indicated that basal-like tumors exhibited the highest expression level of Erk5 transcript among all subtypes, i.e., luminal and HER2-positive subgroups (Fig. 2A). Triplenegative molecular subtyping is routinely utilized in the clinic as a surrogate classification for aggressive basal-like tumors lacking ER/PR and HER2 expression. We found a robust inverse correlation between the level of ERK5 expression and metastasis-free survival of TNBC patients (hazard ratio $=6.9, p$ value $=0.03$; Fig. $2 \mathrm{~B}$ ).

We subsequently analyzed by immunohistochemistry biopsies of human triple-negative ductal carcinoma and adjacent normal breast tissue, as well as lung metastatic sites. In normal breast and lung tissues, ERK5 was specifically detected in acini, ducts and alveoli (Fig. 2C and Supplementary Fig. S1). Higher magnifications clearly demonstrated a predominant ERK5 staining in the cytoplasm of mammary acinar cells. Unlike normal tissue, highgrade invasive ductal carcinoma appeared highly disorganized. The notable absence of tubule formation was associated with the presence of numerous pleomorphic cells exhibiting increased mitotic activity (Fig. 2C and Supplementary Fig. S1). Nonetheless, ERK5 remained predominantly cytoplasmic in cancerous cells with some evidence of perinuclear staining, suggesting that ERK5 was not constitutively activated in triple-negative ductal carcinoma (Fig. 2C and Supplementary Fig. S1). A similar pattern of ERK5 staining was detected in metastatic breast cancer cells from lung biopsies (Supplementary Fig. S1).

Moreover, we confirmed that the protein abundance of ERK5 was higher in TNBC cell lines (MDA-MB-231 and MDA-MB-468) compared with HER2-positive breast cancer cells (BT474, SK-BR-3 and MDA-MB-453) (Supplementary Fig. S2A). Remarkably, ERK5 expressed in TNBC cells did not exhibit the typical mobility shift by SDSpolyacrylamide gel electrophoresis (SDS-PAGE) characteristic of ERK5 activation by hyperphosphorylation 
Fig. 1 ERK5 expression strongly associates with metastatic occurrence in breast cancer. The relationship between the level of ERK5 expression and distant metastasis-free survival of breast cancer $(\mathrm{BC})$ patients was assessed by Kaplan-Meier plotter. Independent cohorts of patients with early (grade 1) or late (grades 2 and 3 ) stage disease status were analyzed. Samples were divided into two groups with high (red) and low (black) expression of ERK5. Hazard ratios (HR) and logrank $P$ values are shown.

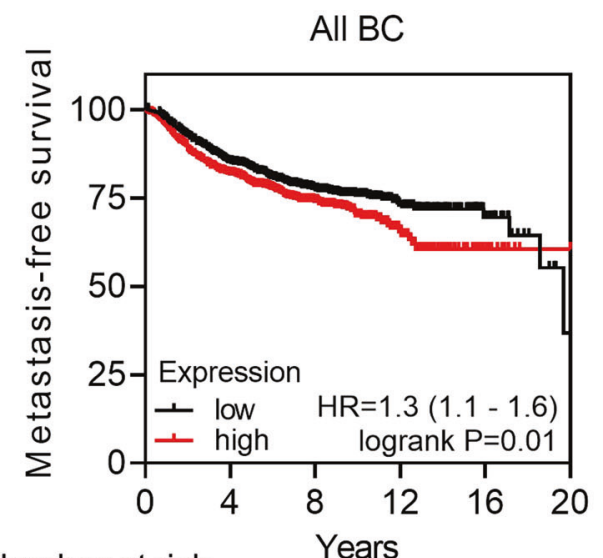

\begin{tabular}{lrlllrrr}
\multicolumn{1}{c}{ Number at risk } & \multicolumn{5}{c}{ Years } \\
low & 1007 & 264 & 361 & 256 & 107 & 27 \\
high & 796 & 241 & 302 & 198 & 55 & 11
\end{tabular}

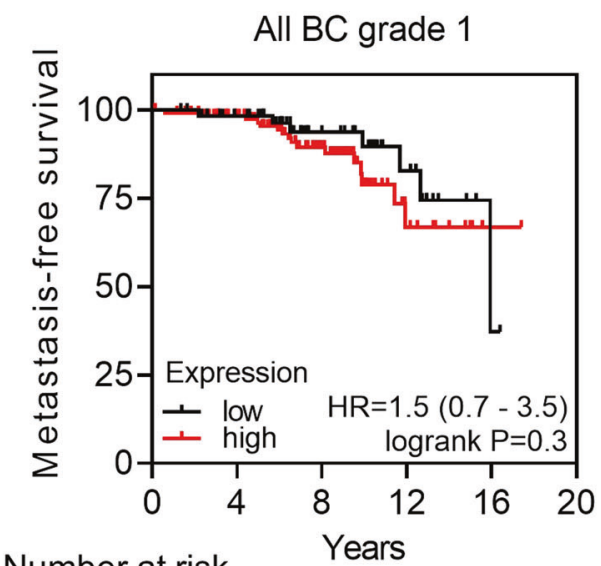

Number at risk

$\begin{array}{lrrrrrr}\text { low } & 62 & 9 & 26 & 15 & 11 & 1 \\ \text { high } & 126 & 20 & 50 & 46 & 9 & 1\end{array}$

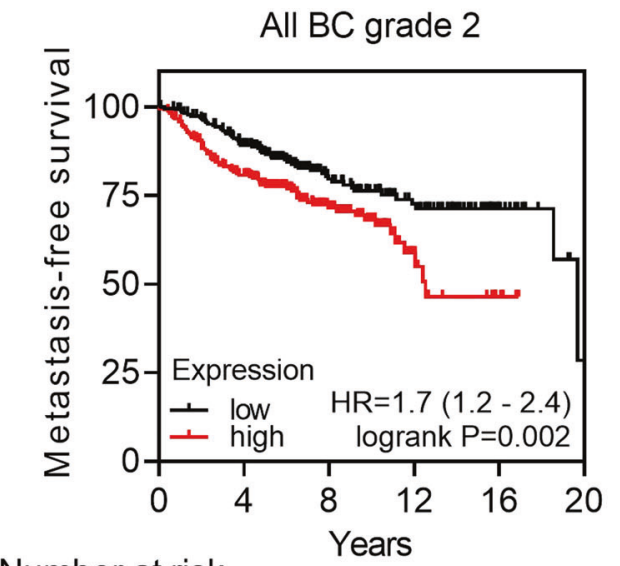

Number at risk

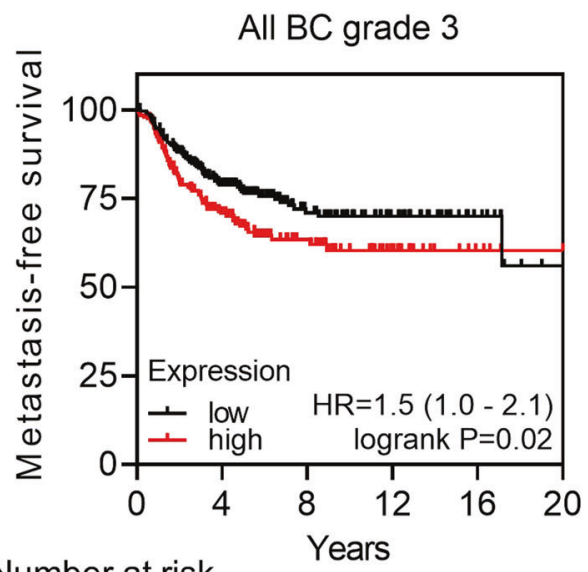

$\begin{array}{llllllr}\text { low } & 276 & 61 & 116 & 44 & 41 & 13 \\ \text { high } & 271 & 76 & 111 & 67 & 12 & 4\end{array}$

Number at risk

$\begin{array}{lrrrrrr}\text { low } & 255 & 50 & 10 & 1 & 0 & 1 \\ \text { high } & 67 & 55 & 9 & 2 & 0 & 0\end{array}$

downstream of HER2 stimulation [19]. The absence of ERK5 hyperphosphorylation in TNBC cell lines was consistent with the lack of nuclear ERK5 staining in sections of human triple-negative breast carcinoma.

\section{ERK5 is required for breast cancer cell invasion and the suppression of E-cadherin expression}

To gain further insights into the oncogenic function of ERK5 in breast cancer, we engineered stable ERK5 knockdown in MDA-MB-231 cells using two distinct shRNAs targeting the $3^{\prime}$ UTR or the coding sequence (CDS) of ERK5. MDA-MB-231 cells transduced with non-target scrambled (Scr) shRNA were utilized as controls. The efficacy of both shRNA to silence ERK5 expression was demonstrated by immunoblot analysis (Fig. 3A, B).

In contrast to ERK5 inhibition by XMD8-92 [20], ERK5 knockdown did not prevent the growth of MDA-MB-231 cells (Supplementary Fig. S2B). Instead, we found that ERK5 silencing significantly impaired breast cancer cell invasion through the Matrigel under fetal bovine serum (FBS)-free condition or in response to EGF treatment, but not following FBS stimulation (Fig. 3C and Supplementary Fig. S2C). Importantly. we confirmed that the invasive capability of shERK5(UTR)-expressing MDA-MB-231 cells was restored following doxycycline-induced expression of FLAG-tagged (F) ERK5 wild type (WT) at a level similar to that of the endogenous protein (Fig. 3B, C). Additionally, real-time quantitative IncuCyte $^{\circledR}$ live cell imaging revealed that silencing ERK5 reduced by around two-fold the kinetic of breast cancer cell adhesion to Matrigel-coated plates, independently of increased cell death up until $12 \mathrm{~h}$ after seeding (Fig. 3D and Video 1). Adherent shERK5(UTR)-expressing MDA-MB-231 cells detected at the end of the experiment exhibited a morphology similar to that of control shScr cell line (Video 1). 
Fig. 2 ERK5 is overexpressed in TNBC and its overexpression strongly correlates with poor prognosis. A The level of Erk5 transcript was analyzed in 416 luminal/HER2 + tumors and 98 TNBC from TCGA dataset.

Black lines in each group indicate median with interquartile range. $\mathbf{B}$

Kaplan-Meier analysis of distant metastasis-free survival of TNBC patients. Samples were divided into two groups with high (red) and low (black) expression of ERK5. Hazard ratio (HR) and logrank $P$ values are shown. C A biopsy of human invasive ductal carcinoma and adjacent normal breast tissue (patient \#481) was stained with $\mathrm{H} \& \mathrm{E}$ or with a specific antibody to ERK5 (brown). Scale bars: (2.5x) $500 \mu \mathrm{m},(10 \mathrm{x}) 100 \mu \mathrm{m},(40 \mathrm{x})$ $20 \mu \mathrm{m}$.
A

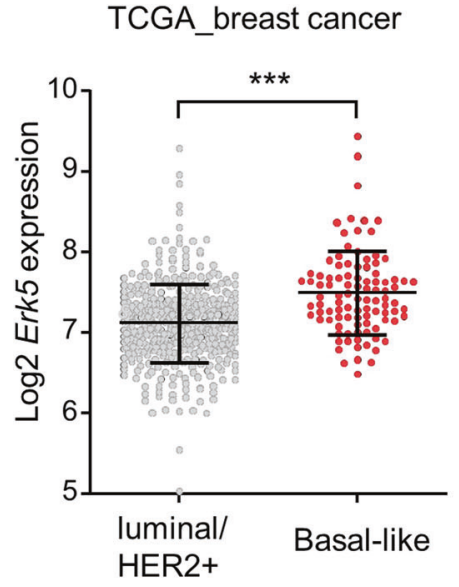

B

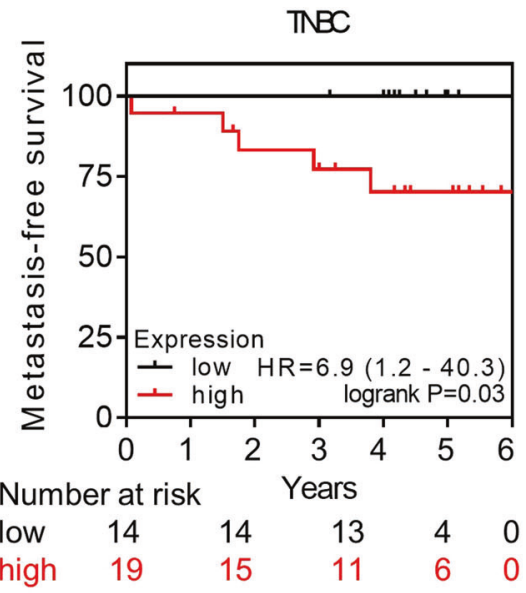

C

Normal breast tissue
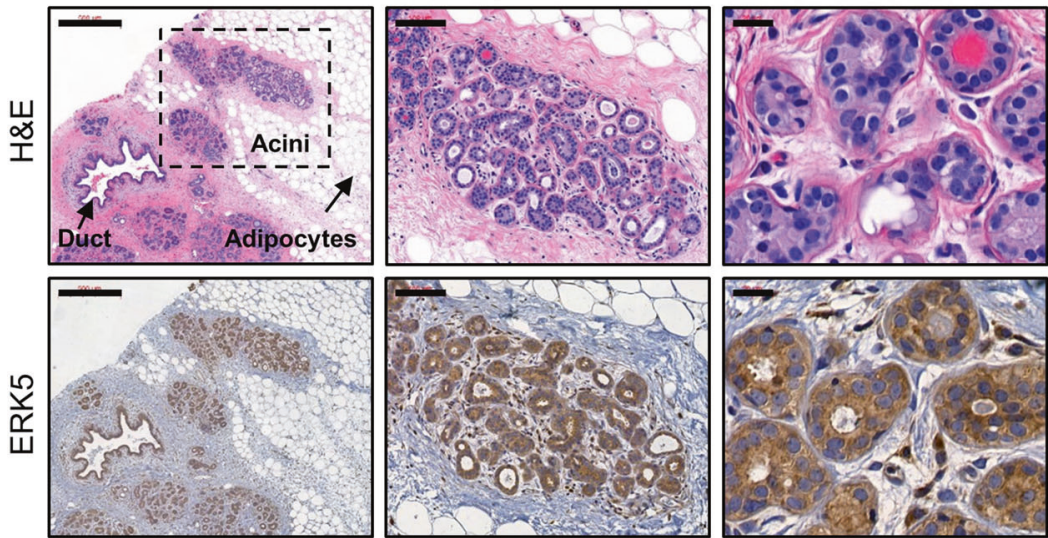

Invasive ductal carcinoma
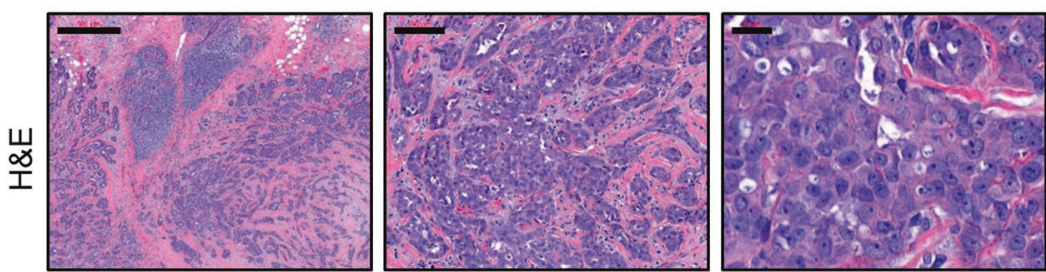

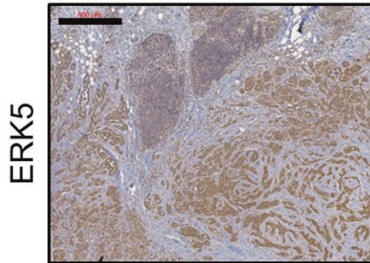

$2.5 x$

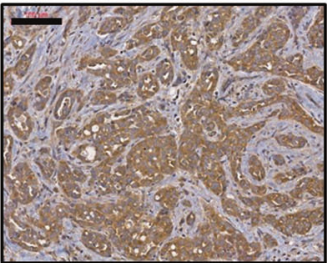

$10 x$

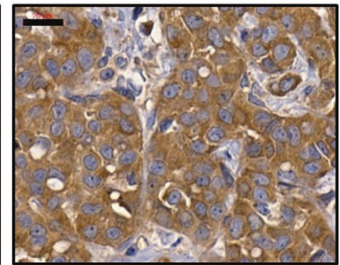

$40 x$
The reduced invasive capability of MDA-MB-231 cells caused by ERK5 silencing correlated with increased expression of the epithelial marker E-cadherin (Fig. 3E). Although the level of the E-cadherin transcript was not significantly altered, this coincided with a marked reduction in the expression of Slug, a transcriptional repressor of E- cadherin involved in EMT (Fig. 3E, F). Conversely, protein and RNA levels of Snail, another important transcriptional repressor of E-cadherin, were increased in shERK5(UTR)compared with shScr-expressing MDA-MB-231 cells, while the transcript level of other mesenchymal markers (e.g., $\mathrm{N}$-cadherin, MMP2, Vimentin) was unchanged (Fig. 3F). 
Fig. 3 ERK5 silencing suppresses breast cancer cell invasion. A, B Immunoblot analysis confirmed that shERK5 (UTR) and shERK5(CDS) effectively downregulated ERK5 expression in MDA-MB231 cells. Ectopic expression of ERK5 [+ F-ERK5(WT)] at a level similar to that of the endogenous protein was achieved by incubating shERK5 (UTR)-expressing cells carrying an inducible F-ERK5(WT) construct with $10 \mathrm{ng} / \mathrm{ml}$ doxycycline (+) for $24 \mathrm{~h}$. C ERK5 knockdown suppressed MDA-MB-231 cell invasion in FBS-free medium through Matrigel. The bar graph indicates the mean $\pm \mathrm{SD}(N=3)$. Representative pictures of the filters are shown. Scale bar: $200 \mu \mathrm{m}$. D Quantitative time course analysis of MDA-MB231 cell adhesion on Matrigel by IncuCyte ${ }^{\circledR}$ live-cell imaging system. Representative pictures of cells taken $4 \mathrm{~h}$ after seeding in Matrigel-coated plates are shown. The data correspond to the mean $\pm \mathrm{SD}(N=3)$. $\mathbf{E}$ Lysates were obtained from cells cultured in FBS-free Matrigelcoated plates for $12 \mathrm{~h}$ and analyzed by immunoblot. The images are representative of three biological repeats. $\mathbf{F}$ Alternatively, transcript levels were measured by quantitative real-time PCR. Results are presented as fold change $\pm S D$. The graph is representative of three independent biological repeats performed in duplicate.
A

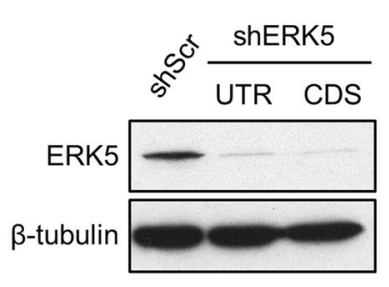

C
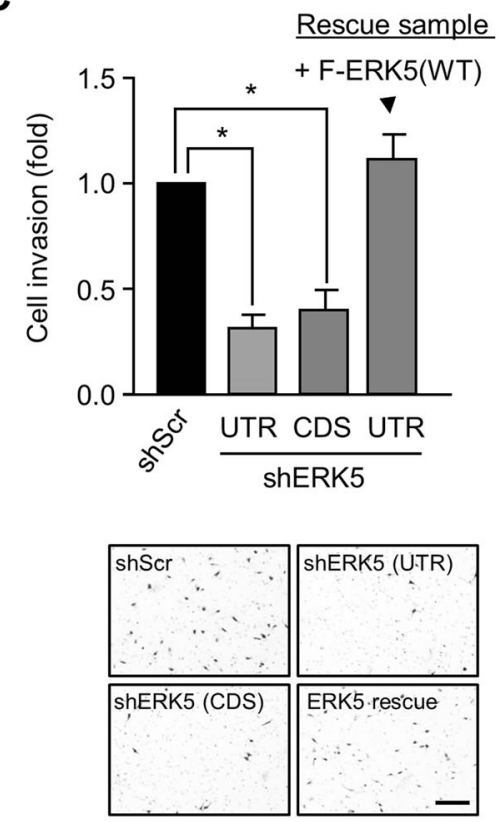

E

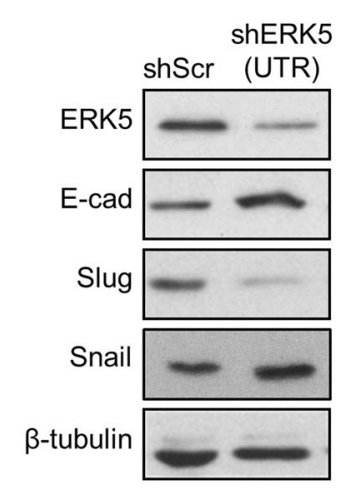

$\mathbf{F}$
B

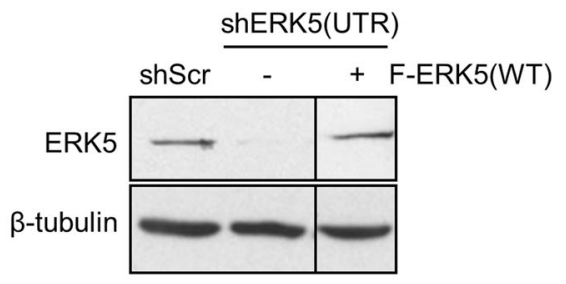

D

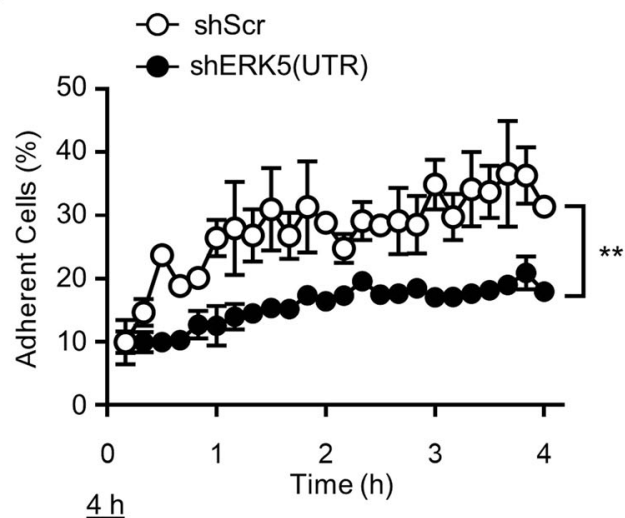

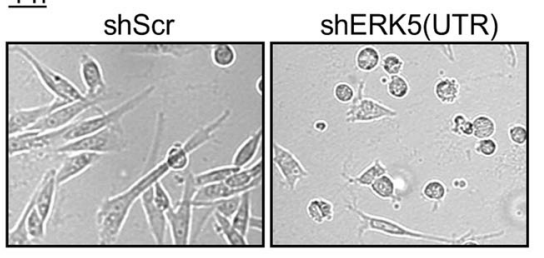

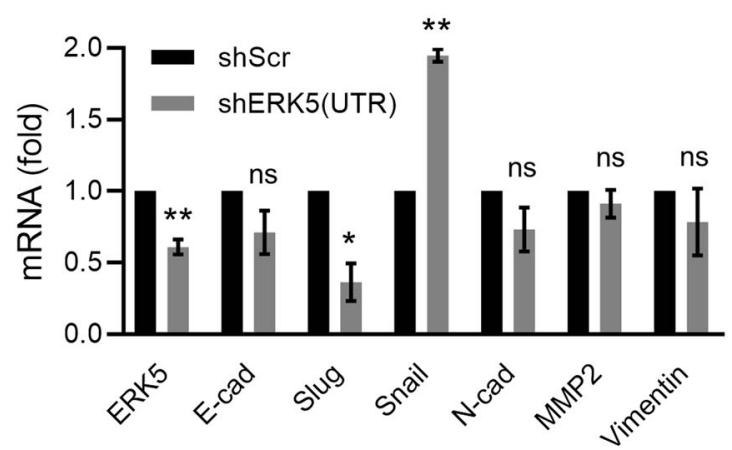

\section{FAK is a potentially important regulator of breast cancer metastasis controlled by a kinase- independent function of ERK5}

To further explore the underlying mechanism of ERK5mediated breast cancer cell invasion, we tested the association between ERK5 and FAK, a non-receptor protein tyrosine kinase that plays a prominent role in cell spreading and migration, and the initiation of various malignancies [21]. The best-characterized mechanism that promotes FAK activation upon cell interactions with the extracellular matrix involves FAK dimerization and subsequent autophosphorylation at Tyr397 [22]. Interestingly, ERK5mediated FAK phosphorylation at Ser910 during melanoma cell migration coincided with decreased phosphorylation at Tyr397 [17].

Here, we found that a proportion of ectopically expressed F-ERK5(WT) in shERK5(UTR)-expressing MDA-MB-231 cells co-localized with phosphorylated FAK at Tyr397 in focal adhesion sites (Fig. 4). Three-dimensional confocal microscopy clearly showed dual staining of F-ERK5(WT) and phospho (p)-FAK(Y397) at cell surface attachment 
Fig. 4 ERK5 co-localizes with p-FAK in focal adhesions. iRFP720 ${ }^{+}$MDA-MB-231 cells carrying shERK5(UTR) were incubated for $24 \mathrm{~h}$ with $2 \mu \mathrm{g} / \mathrm{ml}$ doxycycline to induce F-ERK5 (WT) expression, prior to being seeded on glass bottom dish coated with Matrigel and cultured for $6 \mathrm{~h}$ in FBScontaining medium. Ectopically expressed F-ERK5 and endogenous FAK were visualized by confocal microscopy using specific antibodies to the FLAG epitope (M2, green) and to p-FAK (Y397) (red). Nuclei were detected with DAPI (blue). Images at different $\mathrm{Z}$ positions are shown. Scale bars: $10 \mu \mathrm{m}$.

The inset shows a higher magnification of focal adhesions where F-ERK5 and p-FAK (Y397) co-localized. Violet arrows highlight yellow staining indicative of F-ERK5/p-FAK (Y397) co-localization.

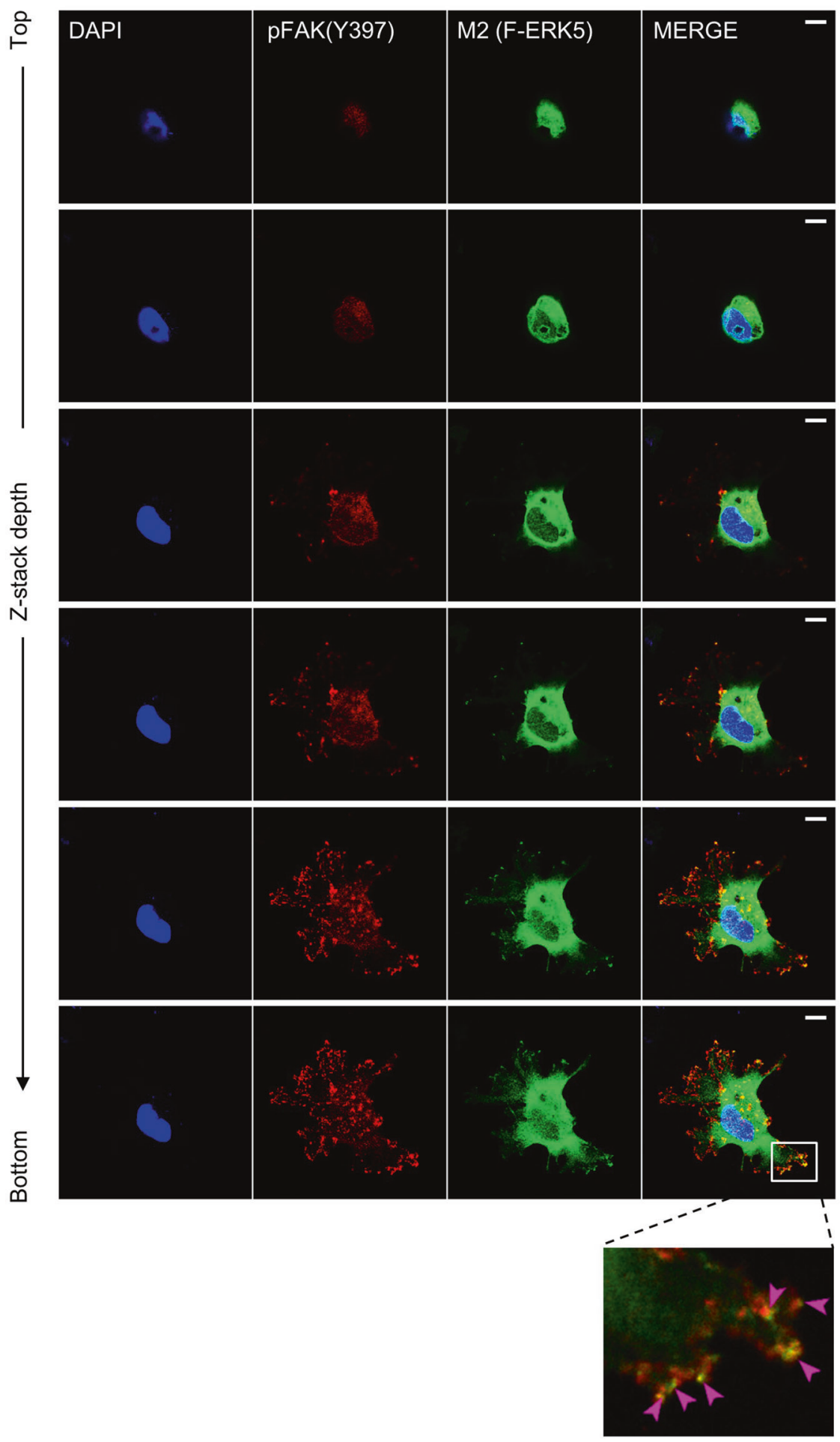

points with the Matrigel (Video 2). We subsequently confirmed by in situ proximity ligation assay (PLA) that F-ERK5(WT) could be detected in very close proximity with FAK, indicative of the existence of an ERK5-FAK complex (Fig. 5A). The specificity of this interaction was supported by evidence that a C-terminal truncated ERK5 mutant [F-ERK5- $\Delta \mathrm{C}(1-575)]$ displaying a predominant nuclear localization [23] did not associate with FAK (Supplementary Fig. S3A).

In parallel, we discovered that ERK5 silencing in breast cancer cells inhibited FAK phosphorylation at Tyr397 (Fig. 5B). The demonstration that doxycycline-induced expression of F-ERK5(WT) restored normal level of FAK phosphorylation in shERK5(UTR)-expressing MDA-MB-231 
Fig. 5 ERK5 interacts with FAK signaling. iRFP720 $0^{+}$ MDA-MB-231 cells carrying shERK5(UTR) were incubated for $24 \mathrm{~h}$ with $2 \mu \mathrm{g} / \mathrm{ml}$ doxycycline to induce F-ERK5 (WT) (A) or F-ERK5(D200A) (D) expression. Cells were subsequently seeded on Matrigel-coated glass bottom plate for $6 \mathrm{~h}$ prior to being fixed and incubated with antibodies to the FLAG epitope (M2) and p-FAK(Y397). Negative controls included incubation with each primary antibody separately and no primary antibodies. In situ detection of ERK5-FAK complexes was performed with oligonucleotidelabeled secondary antibodies according to the Duolink ${ }^{\oplus}$ PLA fluorescence protocol (Sigma). Images were acquired by fluorescence microscopy. Scale bars: $10 \mu \mathrm{m}$. B, C Lysates were obtained from cells cultured in FBS-free Matrigel-coated plates for the indicated times. In panel C, iRFP720 ${ }^{+}$cells expressing shERK5(UTR) were incubated with $10 \mathrm{ng} / \mathrm{ml}$ doxycycline for $24 \mathrm{~h}$ to induce F-ERK5(WT) or F-ERK5(D200A) expression, prior to being seeded. MDAMB-231 cells expressing shScr control or transduced with an empty pCHD plasmid (-) were utilized as controls. Similar results were obtained in two independent experiments.
A

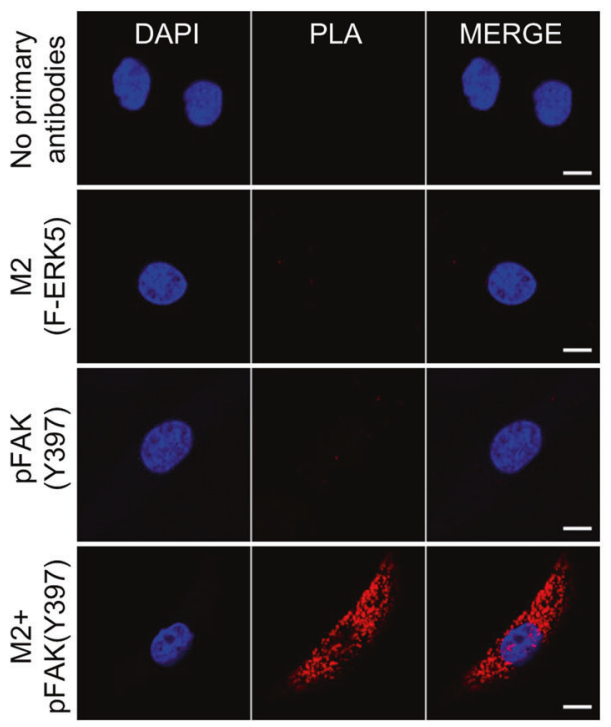

B

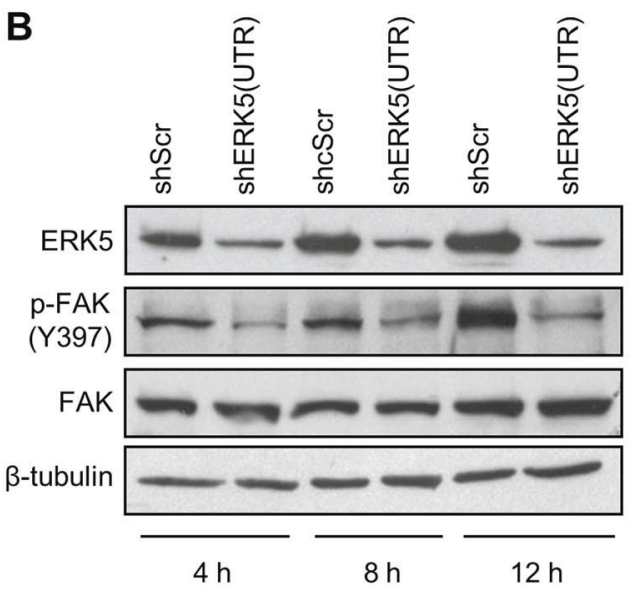

D

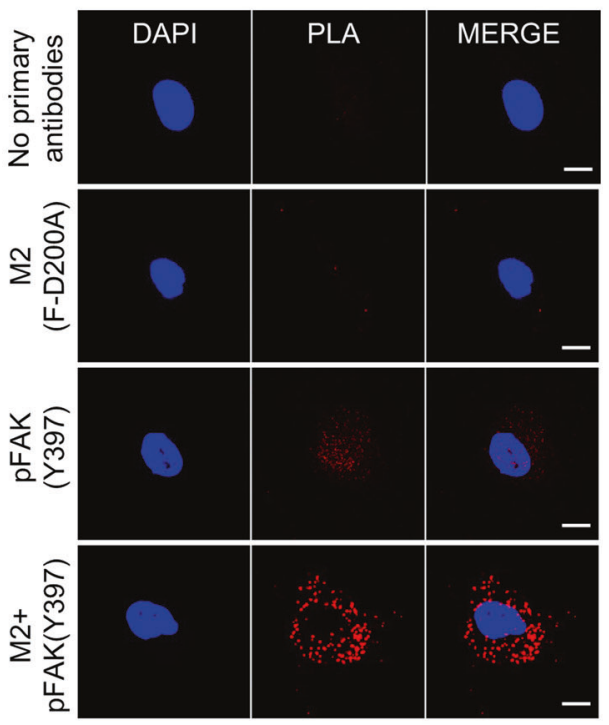

C

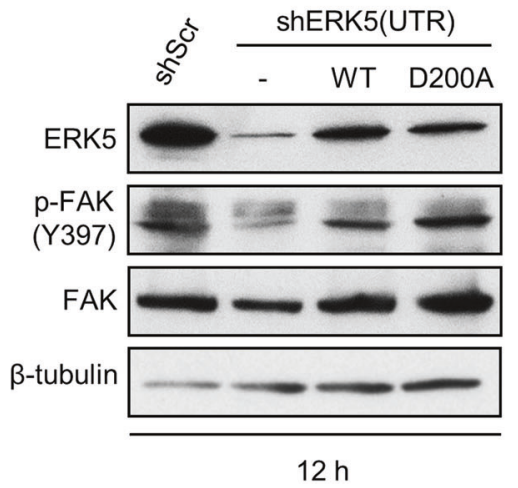

cells confirmed that ERK5 was required for FAK activation in response to signals from the extracellular matrix (Fig. 5C). Remarkably, normal FAK phosphorylation in shERK5 (UTR)-expressing MDA-MB-231 cells was also achieved following induced expression of F-ERK5(D200A), a kinase dead mutant of ERK5 unable to bind ATP [24] (Fig. 5C). We confirmed that JWG-045, a novel pharmacological inhibitor of ERK5 exhibiting significantly reduced affinity for BRD4 compared with XMD8-92 [25, 26], did not suppress FAK phosphorylation at Tyr397 in MDA-MB-231 cells (Supplementary Fig. S3B, C). Interestingly, neither ERK5 silencing nor incubation with JWG-045 affected the level of FAK phosphorylation at Ser910 (Supplementary Fig. S3C). Collectively, these results demonstrated that ERK5 controlled the post-translational modification of FAK at Tyr397, independently of its catalytic activity. Accordingly, F-ERK5(D200A) was able to associate with FAK
(Fig. 5D) and also to rescue the invasion defect caused by ERK5 silencing (Supplementary Fig. S3D, E).

The functional importance of FAK activation downstream of ERK5 was demonstrated by evidence that overexpression of tyrosine phosphorylated FAK at the cell membrane [myristoylated (myr)FAK; [27] rescued the invasion defect caused by ERK5 silencing (Supplementary Fig. S3D, E). Additionally, we confirmed the clinical significance of FAK in TNBC (Supplementary Fig. S4A-C). Consistent with evidence that FAK signaling inversely correlated with metastasis-free survival, inhibiting FAK activity by incubating MDA-MB-231 cells with PND1186 [28] significantly decreased their ability to invade through the Matrigel (Supplementary Fig. S4D). However, PND1186 did not affect the level of ERK5 expression and ERK1/2 phosphorylation (Supplementary Fig. S4E). Likewise, FAK inhibition did not affect the expression of E- 
cadherin, indicating that FAK was not involved in maintaining the mesenchymal phenotype of MDA-MB-231 cells downstream of ERK5 (Supplementary Fig. S4E).

\section{ERK5 is a critical component of malignant progression of breast cancer through FAK phosphorylation}

To confirm the requirement of the ERK5-FAK axis in promoting breast cancer metastasis, we performed tumorigenesis assays in nude mice. First, we created a novel stable MDA-MB-231 cell line to permit doxycyclineinducible ERK5 knockdown in vivo (Supplementary Fig. S5A). MDA-MB-231 cells carrying the shERK5iLuc2 $=$ tdT construct were transplanted into the mammary fat pad of athymic female CD1 mice to allow orthotopic tumor formation. Animals exhibiting small tumors (between 10 and $80 \mathrm{~mm}^{3}$ at day 20) were subsequently fed with a doxycycline containing diet (dox+) to silence ERK5 in breast cancer cells, or with a standard doxycycline-free (dox-) control diet. The presence of doxycycline in the serum of dox + mice was confirmed by using massspectrometry analysis of terminal cardiac blood puncture samples (Supplementary Fig. S5B).

Initially, ERK5 silencing appeared to cause a slight decrease in tumor growth (Fig. 6A). However, no significant difference in tumor size was measured until the absence of ERK5 begun to noticeably accelerate the growth of orthotopic mammary grafts in mice exposed to doxycycline for 3.5 weeks, corresponding to 44 days post transplantation (Fig. 6B). A similar impact of ERK5 silencing on tumor growth was observed in a preliminary small pilot experiment in which MDA-MB-231 cells carrying the shERK5i-Luc2 $=$ tdT construct were transplanted subcutaneously into the back of adult female CBA nude mice (Supplementary Fig. S6A, B). Immunostaining of harvested tumor sections confirmed efficient silencing of ERK5 in orthotopic mammary grafts excised from dox $+\mathrm{CD} 1$ mice (Fig. 6C). Remarkably, the reduction in ERK5 expression correlated with decreased p-FAK(Tyr397) staining, consistent with the requirement of ERK5 for mediating FAK activation in vivo (Fig. 6C). Accordingly, human invasive ductal carcinoma biopsies exhibiting high ERK5 expression displayed high level of p-FAK(Tyr397) (Supplementary Fig. S7).

Mice cohorts were subsequently organized in pairs of dox + and dox - animals according to similar size of tumor volumes (Fig. 7A). The presence of tdT + metastatic breast cancer cells in the lungs of mice carrying orthotopic mammary tumor grafts was quantified post-mortem by flow cytometry (Fig. 7A, B). Consistent with the requirement of ERK5 for mediating breast cancer cell invasion in vitro, the absence of ERK5 notably suppressed breast cancer lung metastasis in vivo. The remaining low incidence of lung metastasis detected in the dox + group was likely a consequence of residual ERK5 level in shERK5i-expressing MDA-MB-231 cells that would be expected in any silencing approach by means of inducible shRNA. Breast cancer cell metastasis to the spleen and the bone was also captured by bioluminescence imaging in one control animal among all recipient mice analyzed (Fig. 7C).

\section{Discussion}

We have demonstrated for the first time that induced ERK5 silencing in small TNBC grafts accelerated the dynamic of tumor growth, suggesting that ERK5 could antagonize mitogenic signaling to maintain cancer cell survival in established tumors. This hypothesis contradicts previous reports that demonstrated that ERK5 knockdown did not affect the growth of primary triple-negative mammary tumors [8-10, 13]. Moreover, a recent publication provided new genetic evidence supporting the requirement of ERK5 in promoting tumorigenesis [29]. These conflicting findings highlight that continued efforts need to be made to resolve the ongoing controversy about the involvement of ERK5 in tumor growth during the early stage development of breast cancer. Nevertheless, ERK5 remains a clinically relevant therapeutic target in TNBC given its strong association with increased metastatic risk (8-10, 29, this study).

Disruption of the actin cytoskeleton by Src through ERK5-dependent transcription constituted the first evidence that ERK5 signaling altered the invasiveness of transformed cells [30, 31]. Here, we proposed that ERK5 maintained the invasive capability of breast cancer cells through FAKmediated regulation of cell adhesion. Accordingly, we provided the first demonstration that decreased malignancy caused by ERK5 ablation associated with the loss of FAK phosphorylation at Tyr397 in mammary tumor grafts. Given the functional interaction between of Src and FAK in focal adhesion remodeling [32], this new finding emphasized a potentially important a role of ERK5 in metastatic mammary tumors with elevated c-Src kinase activity. An alternative mechanism underlying the metastatic effect of ERK5 involved the breast tumor kinase (Brk) downstream of the MET receptor [33, 34]. This might also be significant given that Brk is overexpressed in a majority of breast tumors, whilst undetectable in normal mammary gland [35].

Remarkably, ERK5-mediated FAK phosphorylation at Tyr397 in TNBC cells did not require ERK5 activity, suggesting that ERK5 could act as a scaffold protein to support cell invasion through FAK. Interestingly, ERK5 was mostly detected in the cytoplasmic compartment of cancerous cells in TNBC patients, consistent with the idea that ERK5 overexpression rather than hyperactivation is a 
Fig. 6 Induced ERK5 silencing accelerates tumor growth and suppresses FAK

phosphorylation in mammary tumor grafts. MDA-MB-231 cells carrying shERK5i were orthotopically transplanted into the mammary fad pad of CD1 nude mice. A Representative bioluminescence images of mammary tumors just before (day 20) and after mice were fed with doxycycline for 12 and 22 days. B Tumors were measured on average twice a week for the duration of the experiment. The data presented as fold increase in the volume of tumor size after the introduction of the dox diet at day 20 correspond to the mean $\pm \mathrm{SD}$. After 4 weeks, animals in the dox - and dox + cohorts exhibiting large tumors or a change in normal behavior were humanely culled. Total $N$ number of animals analyzed per condition over the duration of the experiment is indicated. C Tumors were collected 4 weeks after exposure to doxycycline and analyzed by immunohistochemistry with a specific antibody to ERK5 or to p-FAK(Y397). Scale bars: (40x) $20 \mu \mathrm{m}$.
A

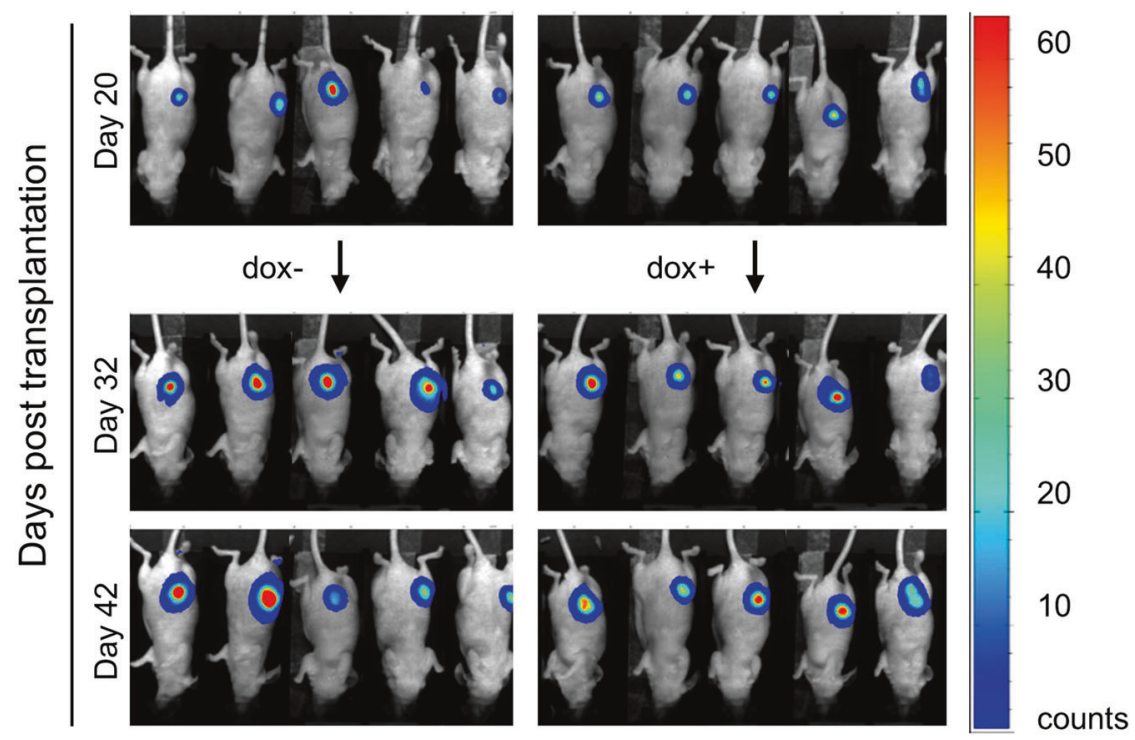

B

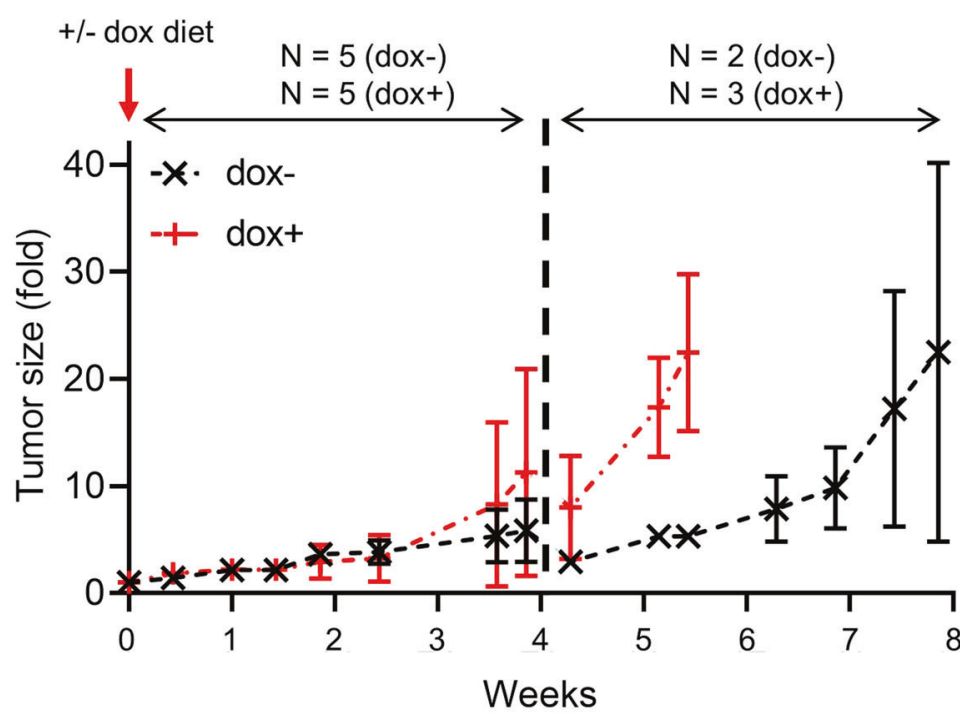

Range:0-229

Min: 0.898

Max:63.8
C

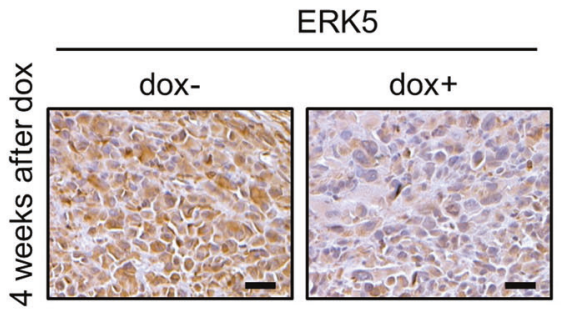

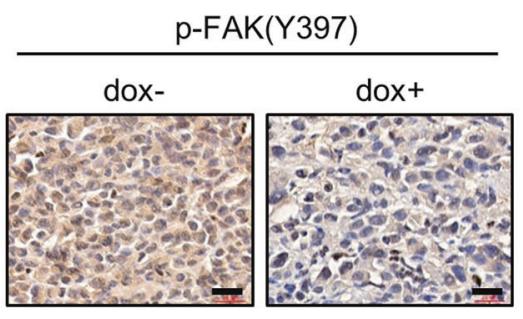

significant feature of high-grade triple-negative invasive ductal carcinoma in humans. Conversely, ERK5 silencing did not affect FAK phosphorylation at Ser910, a site previously found to be phosphorylated by ERK1/2 [36], but also by ERK5 [17-19]. Increased FAK phosphorylation at Ser910 in response to ERK5 activation by MEK5 constituted an important mechanism for mediating melanoma lung metastasis [19]. Interestingly, high level of ERK5 and phosphorylated FAK at Ser910 in highly metastatic murine melanoma and human lung cancer cell lines coincided with low FAK phosphorylation at Tyr397 [19]. A dual function of ERK5 as a scaffold protein (our results) and an enzyme [17-19] might explain these apparent conflicting results. 
Fig. 7 Induced ERK5 silencing suppresses breast cancer lung metastasis. Control (dox-) and doxycycline-fed (dox +$)$ mice bearing mammary tumor grafts were paired according to similar tumor size. A, B Breast metastasis was detected post mortem by quantification of live $\left(\mathrm{DAPI}^{-}\right.$) breast cancer cells $\left(\operatorname{tdT}^{+}\right)$in the lung. A representative flow cytometry analysis is shown in $\mathbf{B}$. The graphical analysis of the data demonstrated that ERK5 knockdown significantly reduced metastatic burden. Ratio paired $t$ test was applied for $p$ value calculation, $p \leq 0.01$ (**). Raw numbers utilized for the graph are presented in the table. C Breast metastasis to the spleen and bone were detected by bioluminescence imaging in one control (dox-) animal of a pair sacrificed at 4 weeks after dox exposure.
A

B

\begin{tabular}{|c|c|c|c|}
\hline Pair \# & $\begin{array}{c}\text { Weeks after } \\
\text { dox exposure } \\
\text { dox-/dox+ }\end{array}$ & dox- & dox+ \\
\hline 1 & $4 / 4$ & 15 & 0 \\
\hline 2 & $4 / 4$ & 4 & 0 \\
\hline 3 & $4 / 5.4$ & 292 & 93 \\
\hline 4 & $13 / 13$ & 228 & 112 \\
\hline 5 & $13 / 13$ & 2957 & 416 \\
\hline
\end{tabular}

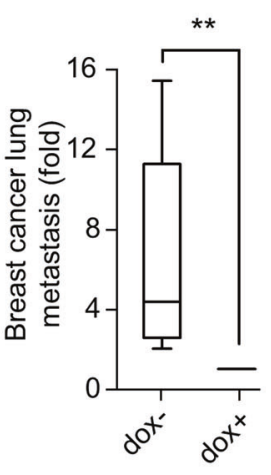

C

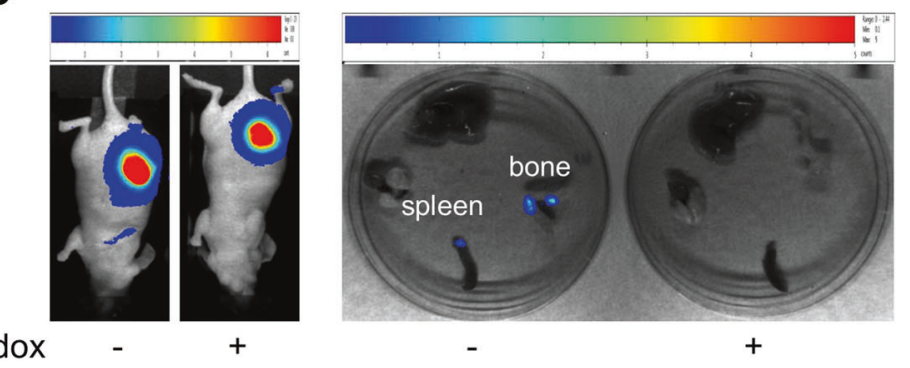

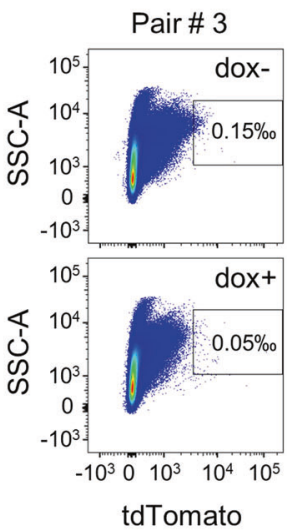

tdTomato
In line with this idea, the estrogen receptor (ER) $\alpha$ was found to suppress breast cancer cell motility and invasion by recruiting ERK5 to the nucleus, thereby restricting the formation of an ERK5/cofilin complex in actin-rich regions of the cytoplasmic membrane [37]. Although the paper did not rigorously address whether or not ERK5 promoted the aggressiveness of $\operatorname{ER} \alpha(-)$ breast cancer cells through modulating actin dynamics independently of its catalytic activity, estrogen-mediated transcription in $\operatorname{ER} \alpha(+)$ breast cancer cells required ERK5 activation by MEK5 [38]. Interestingly, ectopic expression of MEK5 had been previously shown to promote a more malignant phenotype by repressing $\mathrm{ER} \alpha$ expression and by promoting epithelial mesenchymal transition (EMT), a well-known reprograming process that confers metastatic properties to cancer cells [38-40].

Through genetic ablation and silencing approaches, ERK5 was also shown to be essential for maintaining mesenchymal features of MDA-MB-231 tumor grafts $[9,10,29]$. We confirmed that ERK5 suppressed the level of the epithelial marker E-cadherin downstream of Slug. This was not observed in PND1186-treated cells, suggesting that the impact of ERK5 silencing on E-cadherin expression was independent of impaired FAK signaling. Consistent with the CRISPR/Cas9 knock-out approach [29], ERK5 silencing increased the level of Snail. In contrast, we found no evidence that ERK5 altered the level of various mesenchymal markers, e.g., $\mathrm{N}$-cadherin, vimentin, or expression of MMP2, a member of the MMP family known to potentiate cancer cell dissemination through degrading ECM. Moreover, the downregulation of ERK5 did not prevent adherent MDA-MB-231 cells from acquiring the typical stellate morphology characteristic of highly aggressive mesenchymal breast cancer model. These results led us to conclude that, although ERK5 might contribute to controlling the expression level of certain epithelial and mesenchymal markers, the pathological context under which inhibition of the ERK5 pathway is sufficient to reprogram an epithelial phenotype from an aggressive mesenchymal breast cancer model remains unknown.

In summary, we presented strong evidence that ERK5 overexpression was an important feature of TNBC metastasis through the regulation of cell adhesion via FAK. Based on our findings, we hypothesize that ERK5 might contribute to the dimerization of FAK as a prerequisite to FAK activation at focal adhesion sites, via its unique domain structure [23]. In particular, the C-terminal tail of ERK5 that comprises multiple phosphorylation sites, as well as proline-rich sequences, provides ideal binding motifs to serve a scaffolding function of ERK5 in TNBC $[24,41]$. Thus, it will be critical to identify the membraneassociated ERK5 protein complex for understanding the requirement of ERK5 in FAK-mediated metastasis through controlling focal adhesion dynamics. Ultimately, these further investigations might help designing new treatment strategies for aggressive breast cancer based on disrupting 
the scaffolding function of ERK5, rather than inhibiting ERK5 activity.

\section{Materials and methods}

\section{Lentivirus-mediated transfer plasmids}

For constitutive ERK5 knockdown experiments in vitro, shRNA lentiviral transduction particles were purchased from Sigma-Aldrich to target the $3^{\prime} \mathrm{UTR}$ (\#TRCN0000197264) or the CDS (\#TRCN0000010275) of the erk5 transcript. shScr lentiviral particles (\#SHC016V) were utilized as controls. To generate doxycyclinedependent inducible ERK5shRNA, oligonucleotides were designed against the GACCCACCTTTCAGCCTTA sequence at position 5639-5657 in the $3^{\prime}$ UTR of the erk5 gene (Gene ID: 5598). Double-stranded oligonucleotides were subcloned using AgeI and EcoRI in Tet-pLKO-puroIRES-Luc $2=t d T$, a lentiviral transfer plasmid created by inserting a Luc2 $=$ tdT fragment (Addgene \#32904) into the Tet-pLKO-puro vector (Addgene \#21915). Sequences for shERK5i are available in Supplementary Table S1. To construct doxycycline-dependent inducible expression systems, F-ERK5(WT) or D200A mutant cDNAs were subcloned using $P a c \mathrm{I}$ and NheI into the bicistronic lentiviral expression vector pCHD-TRE3GS-MCS-EF1a-iRFP720 (a gift from Stuart Cain, University of Manchester, in which target cDNA expression is driven by the TRE3GS promoter and iRFP720 expression is driven by the EF- $1 \alpha$ core promoter). Sequences for PCR amplification are available in Supplementary Table S1.

\section{Lentiviral infection of MDA-MB-231 cells}

MDA-MB-231 cells were incubated for $24 \mathrm{~h}$ with viral particles at 1 to $2 \mathrm{MOI}$ in the presence of $8 \mu \mathrm{g} / \mathrm{ml}$ polybrene. Infected cells with the pLKO recombinant plasmid were subsequently selected by incubation with $3 \mu \mathrm{g} / \mathrm{ml}$ puromycin until no live cells remained in the non-infected group (at least 3 days). Resistant colonies were pooled and expanded in puromycin-containing medium. Alternatively, MDA-MB-231 cells transduced with doxycycline-inducible F-ERK5(WT) or F-ERK5(D200A) delivering particles for $24 \mathrm{~h}$ were selected by FACS for iRFP720 fluorescence 2 days later.

\section{Transwell invasion assay and measurement of cell adhesion}

For cell invasion, MDA-MB-231 cells were resuspended in FBS-free medium and seeded at a low density of 10,000 cells per well in the upper Boyden chamber of 24-well plates with 8- $\mu \mathrm{m}$ pore (Corning \#353097) coated with Matrigel. The lower chamber was filled with culture medium without FBS. $16 \mathrm{~h}$ after seeding, the cells on the bottom layer surface were stained with $0.05 \%$ crystal violet dye and quantified under the microscope. Cell adhesion in Matrigelcoated plates was monitored by IncuCyte ${ }^{\circledast}$ live cell analysis. Four fields of view were set up for each well. The number of cells in each field was counted in real time, every $10 \mathrm{~min}$. Adherent cells were distinguished from non-adherent cells based on a larger cell surface area. Triplicate data were used for statistical analysis.

\section{Mammary tumor grafts}

Eighteen adult female CD1 nude mice were injected in the mammary fad pad (4th nipple orthotopic) with MDA-MB231 cells carrying the shERK5(UTR-2)-Luc $=$ tdT construct $\left(2 \times 10^{6}\right.$ cells in $50 \mu \mathrm{l}$ PBS mixed with $50 \mu$ l Matrigel $)$. After small tumors were established, 9 mice received doxycycline in their diet, while 9 control animals were fed with a standard doxycycline-free diet. Tumor size was determined by caliper measurements of tumor length, width and depth and expressed as volume in $\mathrm{mm}^{3}(0.52 \times$ length $\times$ width $\times$ depth). Four experimental mice in each cohort exhibited tumors that stopped growing few weeks after the introduction of the dox + or dox - diets. Therefore, 5 animals per groups were utilized for statistical analysis to confirm the effect and rule out artefacts associated with biological variability. In parallel, tumor growth was analyzed by bioluminescence imaging. Mice received an intraperitoneal injection of $100 \mu \mathrm{l}$ of luciferin $(30 \mathrm{mg} / \mathrm{ml})$ and imaged using a bioluminescent imaging system (PhotonImager Opitma, Biospace Lab, France). Images from dox - and dox + group were captured under identical imaging conditions at identical times after luciferin injection.

\section{Analysis of mammary tumor lung metastasis by flow Cytometry}

Metastasis was analyzed post mortem in the lung of mice bearing large mammary tumor grafts (around $700 \sim 800 \mathrm{~mm}^{3}$ ). In brief, lungs were collected, minced in a Petri dish on ice and sequentially digested in Hanks balanced salt solution (HBSS) containing collagenase $(1.25 \mathrm{mg} / \mathrm{ml})$ and hyaluronidase $(125 \mu \mathrm{g} / \mathrm{ml})$ for $30 \mathrm{~min}$, trypsin-EDTA for $3 \mathrm{~min}$, and dispase $(5 \mathrm{mg} / \mathrm{ml})$ and DNase $(10 \mathrm{mg} / \mathrm{ml})$ for $3 \mathrm{~min}$. Neutralization with $1 \%$ FBScontaining HBSS was conducted between each enzymatic digestion, following centrifugation at $5000 \mathrm{rpm}$. The final cell suspension was filtered through a $40 \mu \mathrm{m}$ cell strainer. Red blood cells were solubilized with red cell lysis buffer (Pharm Lyse, BD Biosciences) and the resulting suspension was filtered through a cell strainer to produce single-cell 
suspensions. Dissociated cells were washed twice in PBS and incubated with DAPI (blue). For analysis, a typical forward- and side-scatter gate was set to exclude dead cells and aggregates; a total of $2 \times 106$ events in the gate were collected using the BD Biosciences LSR Fortessa cytometer. tdTomato positive cells were quantified using Flowjo software.

\section{Kaplan-Meier analysis}

Kaplan-Meier plots were generated through the Jetset best probes (dataset number \# '35617_at' for ERK5 and '208820_at' for FAK) to evaluate the prognostic significance of ERK5 and FAK mRNA in distant metastasisfree survival (http://www.kmplot.com). For Fig. 1, patient specimens were divided into high and low expression groups according to the median expression. The median was computed before any subgroups were analyzed. Consequently, the same threshold of ERK5 transcript level was utilized in all panels. The grade subgroup information was determined in the website database. For Figs. 2B, S4A and S4C, patient data were analyzed using the best cutoff to distinguish high and low expression groups. Patients marked as 'ER negative', 'PR negative' and 'HER2 negative' were grouped as TNBC patients. Hazard ratios (HR) with $95 \%$ confidence intervals and logrank $P$ values were calculated automatically by the website software.

\section{Statistical analyses}

Results were analyzed using the two-way ANOVA test to compare time courses. For normally distributed data, an unpaired two-tailed Student's $t$ test was performed. Statistical significance was set at $* p \leq 0.05, * * p \leq 0.01$, *** $p \leq$ 0.001 ; $n s$ indicates no statistical difference. For in vivo analysis of metastatic burden, the number of breast cancer cells extracted from the lung of paired tumor-bearing mice was normalized as ratios $\left(\frac{d o x-}{d o x+}\right.$, when dox + is 0 , calculate it as 1) and compared using a ratio paired $t$ test.

Additional materials and methods are listed in the Supplementary information.

\section{Data availability}

The authors declare that all the data supporting the findings of this study are available within the paper and its supplementary information files. All other data supporting the findings of this study are available from the corresponding authors upon reasonable request.

Acknowledgements This work was supported by a grant from Worldwide Cancer Research to CT and a Confidence in Concept scheme award from the Medical Research Council to KGF. We thank
Peter March and Roger Meadow (Bioimaging), Mike Jackson (Flow Cytometry), George Taylor (Mass Spectrometry), Duncan Forster (Wolfson Molecular Imaging Center), and Peter Walker (Histology) from core research facilities (University of Manchester) for very helpful advice, and the staff at the University of Manchester Biological Safety Unit for looking after the mice. We would also like to thank Andrew Gilmore and Stuart Cain from the University of Manchester for providing essential reagents for the execution of the work.

\section{Compliance with ethical standards}

Conflict of interest The authors declare no competing interests.

Publisher's note Springer Nature remains neutral with regard to jurisdictional claims in published maps and institutional affiliations.

Open Access This article is licensed under a Creative Commons Attribution 4.0 International License, which permits use, sharing, adaptation, distribution and reproduction in any medium or format, as long as you give appropriate credit to the original author(s) and the source, provide a link to the Creative Commons license, and indicate if changes were made. The images or other third party material in this article are included in the article's Creative Commons license, unless indicated otherwise in a credit line to the material. If material is not included in the article's Creative Commons license and your intended use is not permitted by statutory regulation or exceeds the permitted use, you will need to obtain permission directly from the copyright holder. To view a copy of this license, visit http://creativecommons. org/licenses/by/4.0/.

\section{References}

1. Narod SA, Iqbal J, Miller AB. Why have breast cancer mortality rates declined? J Cancer Policy. 2015;5:8-17.

2. Vuong D, Simpson PT, Green B, Cummings MC, Lakhani SR. Molecular classification of breast cancer. Virchows Arch. 2014:465:1-14.

3. Sledge GW, Mamounas EP, Hortobagyi GN, Burstein HJ, Goodwin PJ, Wolff AC. Past, present, and future challenges in breast cancer treatment. J Clin Oncol. 2014;32:1979-86.

4. Montero JC, Ocaña A, Abad M, Ortiz-Ruiz MJ, Pandiella A, Esparís-Ogando A. Expression of Erk5 in early stage breast cancer and association with disease free survival identifies this kinase as potential therapeutic target. PLoS One. 2009;4:e5565.

5. Ortiz-Ruiz MJ, Álvarez-Fernández S, Parrott T, Zaknoen S, Burrows FJ, Ocaña A, et al. Therapeutic potential of ERK5 targeting in triple negative breast cancer. Oncotarget. 2014;5:11308-18.

6. Al-Ejeh F, Miranda M, Shi W, Simpson PT, Song S, Vargas AC, et al. Kinome profiling reveals breast cancer heterogeneity and identifies targeted therapeutic opportunities for triple negative breast cancer. Oncotarget. 2014;5:3145-58.

7. Miranda M, Rozali E, Khanna KK, Al-Ejeh F. MEK5-ERK5 pathway associates with poor survival of breast cancer patients after systemic treatments. Oncoscience. 2015;2:99-101.

8. Cronan MR, Nakamura K, Johnson NL, Granger DA, Cuevas BD, Wang JG, et al. Defining MAP3 kinases required for MDA-MB231 cell tumor growth and metastasis. Oncogene. 2012;31: 3889-900.

9. Javaid S, Zhang J, Smolen GA, Yu M, Wittner BS, Singh A, et al. MAPK7 regulates EMT features and modulates the generation of CTCs. Mol Cancer Res. 2015;13:934-43.

10. Pavan S, Meyer-Schaller N, Diepenbruck M, Kalathur RKR, Saxena M, Christofori G. A kinome-wide high-content siRNA 
screen identifies MEK5-ERK5 signaling as critical for breast cancer cell EMT and metastasis. Oncogene. 2018;37:4197-213.

11. Zhou C, Nitschke AM, Xiong W, Zhang Q, Tang Y, Bloch M, et al. Proteomic analysis of tumor necrosis factor-alpha resistant human breast cancer cells reveals a MEK5/Erk5-mediated epithelial-mesenchymal transition phenotype. Breast Cancer Res. 2008;10:R105.

12. Chen R, Yang Q, Lee JD. BMK1 kinase suppresses epithelialmesenchymal transition through the Akt/GSK3 $\beta$ signaling pathway. Cancer Res. 2012;72:1579-87.

13. Zuo Y, Wu Y, Wehrli B, Chakrabarti S, Chakraborty C. Modulation of ERK5 is a novel mechanism by which Cdc42 regulates migration of breast cancer cells. J Cell Biochem. 2015;116:124-32.

14. Sawhney RS, Liu W, Brattain MG. A novel role of ERK5 in integrin-mediated cell adhesion and motility in cancer cells via Fak signaling. J Cell Physiol. 2009;219:152-61.

15. Villa-Moruzzi E. Targeting of FAK Ser910 by ERK5 and PP1delta in non-stimulated and phorbol ester-stimulated cells. Biochem J. 2007;408:7-18.

16. Ali M, Mutahir Z, Riaz A. CRISPR/Cas9 engineering of ERK5 identifies its FAK/PYK2 dependent role in adhesion-mediated cell survival. Biochem Biophys Res Commun. 2019;513:179-85.

17. Jiang W, Cai F, Xu H, Lu Y, Chen J, Liu J, et al. Extracellular signal regulated kinase 5 promotes cell migration, invasion and lung metastasis in a FAK-dependent manner. Protein Cell. 2020;11:825-45.

18. Cancer Genome Atlas Network. Comprehensive molecular portraits of human breast tumours. Nature. 2012;490:61-70.

19. Esparís-Ogando A, Díaz-Rodríguez E, Montero JC, Yuste L, Crespo P, Pandiella A. Erk5 participates in neuregulin signal transduction and is constitutively active in breast cancer cells overexpressing ErbB2. Mol Cell Biol. 2002;22:270-85.

20. Perez-Madrigal D, Finegan KG, Paramo B, Tournier C. The extracellular-regulated protein kinase 5 (ERK5) promotes cell proliferation through the down-regulation of inhibitors of cyclin dependent protein kinases (CDKs). Cell Signal. 2012;24:2360-8.

21. Sulzmaier FJ, Jean C, Schlaepfer DD. FAK in cancer: mechanistic findings and clinical applications. Nat Rev Cancer. 2014;14:598-610.

22. Brami-Cherrier K, Gervasi N, Arsenieva D, Walkiewicz K, Boutterin MC, Ortega A, et al. FAK dimerization controls its kinasedependent functions at focal adhesions. EMBO J. 2014;33:356-70.

23. Pearson AJ, Fullwood P, Toro Tapia G, Prise I, Smith MP, Xu Q, et al. Discovery of a gatekeeper residue in the C-terminal tail of the extracellular signal-regulated protein kinase 5 (ERK5). Int $\mathbf{J}$ Mol Sci. 2020;21:929.

24. Kasler HG, Victoria J, Duramad O, Winoto A. ERK5 is a novel type of mitogen-activated protein kinase containing a transcriptional activation domain. Mol Cell Biol. 2000;20:8382-9.

25. Williams CA, Fernandez-Alonso R, Wang J, Toth R, Gray NS, Findlay GM. Erk5 is a key regulator of naive-primed transition and embryonic stem cell identity. Cell Rep. 2016;16:1820-8.

26. Wang J, Erazo T, Ferguson FM, Buckley DL, Gomez N, MuñozGuardiola P, et al. Structural and atropisomeric factors governing the selectivity of pyrimido-benzodiazipinones as inhibitors of kinases and bromodomains. ACS Chem Biol. 2018;13:2438-48.

27. Zouq NK, Keeble JA, Lindsay J, Valentijn AJ, Zhang L, Mills D, et al. FAK engages multiple pathways to maintain survival of fibroblasts and epithelia: differential roles for paxillin and p130Cas. J Cell Sci. 2009;122:357-67.

28. Tanjoni I, Walsh C, Uryu S, Tomar A, Nam J-O, Mielgo A, et al. PND-1186 FAK inhibitor selectively promotes tumor cell apoptosis in three-dimensional environments. Cancer Biol Ther. 2010;9:764-77.

29. Hoang VT, Matossian MD, Ucar DA, Elliott S, La J, Wright MK, et al. ERK5 is required for tumor growth and maintenance through regulation of the extracellular matrix in triple negative breast cancer. Front Oncol. 2020;10:1164.

30. Barros JC, Marshall CJ. Activation of either ERK1/2 or ERK5 MAP kinase pathways can lead to disruption of the actin cytoskeleton. J Cell Sci. 2005;118:1663-71.

31. Schramp M, Ying O, Kim TY, Martin GS. ERK5 promotes Srcinduced podosome formation by limiting Rho activation. J Cell Biol. 2008;181:1195-210.

32. Wu JC, Chen YC, Kuo CT, Wenshin Yu H, Chen YQ, Chiou A. et al. Focal adhesion kinase-dependent focal adhesion recruitment of SH2 domains directs SRC into focal adhesions to regulate cell adhesion and migration. Sci Rep. 2015;5:18476

33. Castro NE, Lange CA. Breast tumor kinase and extracellular signal-regulated kinase 5 mediate Met receptor signaling to cell migration in breast cancer cells. Breast Cancer Res. 2010;12:R60.

34. Locatelli A, Lange CA. Met receptors induce Sam68-dependent cell migration by activation of alternate extracellular signal-regulated kinase family members. J Biol Chem. 2011;286:21062-72.

35. Goel RK, Lukong KE. Tracing the footprints of the breast cancer oncogene BRK - Past till present. Biochim Biophys Acta. 2015;1856:39-54.

36. Jiang X, Sinnett-Smith J, Rozengurt E. Differential FAK phosphorylation at Ser-910, Ser-843 and Tyr-397 induced by angiotensin II, LPA and EGF in intestinal epithelial cells. Cell Signal. 2007;19:1000-19.

37. Madak-Erdogan Z, Ventrella R, Petry L, Katzenellenbogen BS. Novel roles for ERK5 and cofilin as critical mediators linking $\mathrm{ER} \alpha$-driven transcription, actin reorganization, and invasiveness in breast cancer. Mol Cancer Res. 2014;12:714-27.

38. Antoon JW, Martin EC, Lai R, Salvo VA, Tang Y, Nitzchke AM, et al. MEK5/ERK5 signaling suppresses estrogen receptor expression and promotes hormone-independent tumorigenesis. PLoS One. 2013;8:e69291.

39. Liu F, Zhang H, Song H. Upregulation of MEK5 by Stat3 promotes breast cancer cell invasion and metastasis. Oncol Rep. 2017;37:83-90.

40. Brabletz, Thomas RaghuKalluri, Nieto MAngela, Weinberg RobertA. EMT in cancer. Nat Rev Cancer. 2018;18:128-34.

41. Nithianandarajah-Jones GN, Wilm B, Goldring CE, Müller J, Cross MJ. ERK5: structure, regulation and function. Cell Signal. 2012;24:2187-96. 